\title{
Scanning-tunneling microscopy at small tip-to-surface distances
}

\author{
S. Ciraci* and Inder P. Batra \\ IBM Almaden Research Center, 650 Harry Road, San Jose, California 95120-6099 \\ (Received 11 May 1987; revised manuscript received 12 August 1987)
}

\begin{abstract}
The scanning-tunneling microscopy (STM) of graphite at small tip-to-surface distances is investigated using the self-consistent-field pseudopotential method. We have calculated potential, charge density in the region between the tip and surface, and the force corrugation. Our results reveal that the tip at the close proximity to the surface disturbs the states near the Fermi level, and induces localized states. The STM images, which are usually related to the local density of states at the Fermi level of the clean surface, are affected by these localized states. The tunneling barrier is shown to collapse at small distances and a new mechanism for current is postulated. Some experimental evidence for this effect is presented.
\end{abstract}

In the scanning-tunneling microscopy ${ }^{1}$ (STM) the distance between the tip and the substrate surface $(h)$ is known to be a critical parameter, but it cannot be precisely measured. The theory of the tunneling developed by Bardeen ${ }^{2}$ assumes a wide and high potential barrier, in which the tails of the substrate and tip wave functions slightly overlap. Based on this theory, Tersoff and Hamann ${ }^{3}$ showed that the STM images the local density states at the Fermi level $\rho\left(\mathbf{r}, E_{F}\right)$. The Tersoff and Hamann ${ }^{3}$ theory has been applied ${ }^{4-6}$ with reasonable success in identifying ${ }^{4,5}$ two distinct atoms on the graphite surface observed by STM. ${ }^{7,8}$ High asymmetry seen between these atoms was explained by Batra et al. ${ }^{5}$ and other graphite images by Mizes and Harrison. ${ }^{6}$ However, the explanation for "giant corrugation" continues to be controversial. ${ }^{9-11}$ Atomic resolution has recently been reported $^{12}$ for graphite using atomic force microscopy (AFM). ${ }^{13}$

Recent STM studies ${ }^{9}$ have indicated the possibility that the elastic deformation due to the close proximity of the tunneling tip to the graphite surface may explain the observed $^{7,8}$ giant corrugation. A tip-to-surface distance, $h$, as small as $2-3 \AA$, is proposed. If this is the case, the effective potential barrier recedes or totally disappears, and the current is expected to have a significant contribution from nontunneling electronic states. In this work, we have investigated such a situation by using a simple model. We have carried out standard self-consistent pseudopotential calculations ${ }^{14}$ in the momentum space ${ }^{15}$ on a system consisting of the graphite surface and the tip with varying positions. Calculations of the electronic states, charge density, and atomic forces reveal important qualitative results: (i) The tip at the close proximity of the surface disturbs the graphite states near the Fermi level induces a nonequilibrium (unstable) chemical bond, or localized states in the vacuum region. These tip-induced states are expected to influence the STM images at constant $V$ and $I$, which are usually related to $\rho\left(\mathbf{r}, E_{F}\right)$. (ii) For small $h$, the repulsive tip force at the $B$ site $^{5,16}$ has large value and large decay constant. It is larger than the tip force at the $H$ site. This situation is, however, reversed when $h$ is increased to 4.7 a.u.

Present results are the first ab initio demonstration of the force corrugation in AFM. The elastic deformation model used to explain the giant corrugation at small bias voltage is based on a similar force concept derived from an empirical potential. ${ }^{9}$ Recently this elastic deformation model has been elucidated by Mamin et al. ${ }^{11}$ The calculation of the local deformation by using the present selfconsistent field method is computationally not feasible, and therefore the giant-corrugation issue is beyond the scope of this study. Nevertheless, our study justifies the role of the tip-induced force in amplifying the chargedensity corrugation.

To study the electronic structure and the total energy we have used a periodic tip within the repeating slab model. The periodic tip is simulated by a single carbon atom with an intertip distance of 4.65 a.u., corresponding to the second-nearest-neighbor distance in graphite. This certainly produces an intertip interaction, which as deduced from the isolated monolayer calculations is, however, small. At large tip-to-surface distance $(h \simeq 8$ a.u. $)$ we realize that the intertip interaction is larger than the tipsurface interaction. Since, the intertip interactions are nearly constant for $2.7<h<8$ a.u. our qualitative conclusions should not be affected by its presence. At small $h$ ( $h=2.7$ a.u., which is comparable to the first nearestneighbor distance in graphite) the desired tip-surface interaction plays a dominant role. This interaction is determined mainly by the outermost atom of the tip. Apparently, the representation of the tip by a single atom is found to be appropriate.

The graphite surface is represented by a three-atomlayer slab with an interslab distance of $1.5 c=18.99$ a.u. ( $c$ being twice the interlayer distance) ${ }^{17}$ Clearly, for large $h$, the interlayer interaction between (0001) planes is weaker than the intertip interaction, which is the artifact of the tip with small periodicity. In spite of that we use a multilayer slab of graphite for the following reasons: The first is to avoid the pathological singularities of the graphite monolayer leading to an infinite corrugation; ${ }^{10}$ the second is the comparison of the interlayer potential barrier with that occurring between the surface and the tip, which provides information about the collapse of the tunneling barrier at small $h$.

Plane waves having kinetic energy less than $10 \mathrm{Ry}$ are treated exactly, and those between 10 and $13 \mathrm{Ry}$ are included via the Lowdin's perturbation scheme. So we had 
a modest number $(-490)$ of plane waves to represent the Bloch states. It is clear that the absolute total energies in our calculations with the 10-13-Ry kinetic-energy cutoff are not converged. ${ }^{18}$ The absolute convergence in the present system requires a much larger basis set which is beyond the current computational capacity. Therefore the calculated total energies should be used only for relative comparisons, and the values of the forces are good only for getting qualitative trends.

Figure 1 illustrates the contour plots of the total charge density and the interatomic forces calculated selfconsistently for the tip being above the $B$ site at three different values of $h$. The potential barrier between the tip and the graphite surface calculated using the planarly averaged [one dimensional (1D)] $l=0$ part of the nonlocal pseudopotential ${ }^{19}$ is also shown for each $h$. For $h=2.7$ a.u. we see that the potential barrier collapses, and a "bond" is set up from the C $2 p_{z}$ orbitals of the tip and
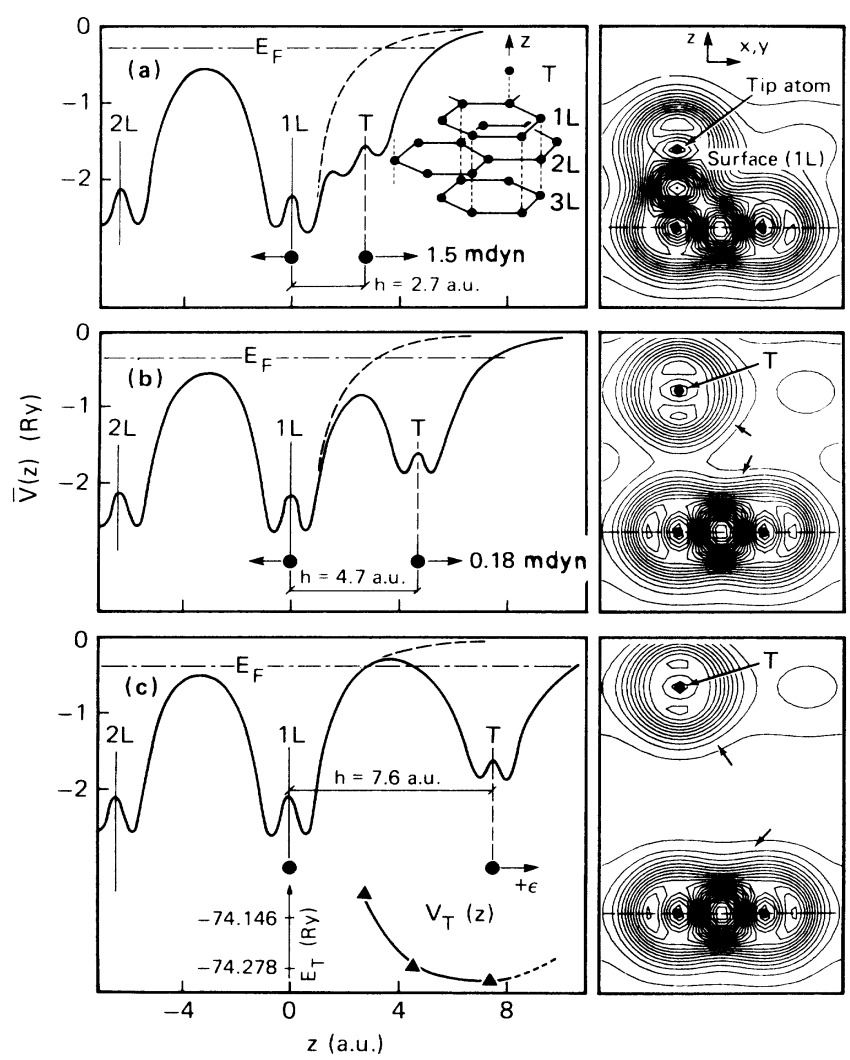

FIG. 1. Planarly average 1D pseudopotential $\bar{V}(z)$, interatomic forces, and contour plots of the total charge density for various tip-to-surface distances $h$. (a) $h=2.7$ a.u., (b) $h=4.7$ a.u., (c) $h=7.6$ a.u. The inset in panel (a) describes the threelayer graphite slab and the carbon atom $(T)$ representing the tip. Total energies for various values of $h, E_{T}$, and the potential energy $V_{T}(h)$ curve obtained therefrom are shown in panel (c). The averaged $1 \mathrm{D}$ potential $\bar{V}(z)$ with and without the tip atom are shown by the solid and dashed lines, respectively. The zero of $\bar{V}(z)$ is set at the vacuum level. The filled circles denote the position of the carbon atoms. The value of the total charge density increases along the direction of the small arrows. nearest surface atoms. The energy of the state associated with this bond, and those associated with $\mathrm{C} 2 p_{x}$ and $2 p_{y}$ orbitals of the tip are located near the Fermi level. Their locations relative to the Fermi level are influenced by the value of $h$. Retracting the tip, and thus increasing $h$, causes the bond (or the localized states) to be delocalized gradually. Finally, the C $2 p_{x}, 2 p_{y}$, and $2 p_{z}$ orbitals of the tip become degenerate when $h$ is much larger than the $\mathrm{C}-\mathrm{C}$ bond distance. Since the interaction between the surface and tip atoms is local, the above description of localization would not be affected even if the tip is represented by a cluster of atoms. We arrive at this conclusion by examining the charge density and the origin of the states near the Fermi level. As also seen from Figs. 1 (b) and 1 (c), upon retracting the tip the height of the potential barrier increases, and rises above the Fermi level. Calculated total energies and tip forces are listed in Table I. For a given kinetic-energy cutoff, the interatomic forces require a very high SCF convergence criterion. ${ }^{20}$ The error bars in Table I arise from the modest SCF convergence criterion (rms deviation in potential) $\sim 5 \times 10^{-5}$ Ry used in the present study.

A strong repulsive force of $\sim 1.5 \mathrm{mdyn}\left(1 \mathrm{mdyn}=10^{-8}\right.$ $\mathrm{N})$ is exerted on the tip for $h=2.7$ a.u. This force is balanced by a resultant reaction force distributed over the layers of the graphite. It is large on the surface layer, but decays rapidly as one goes deeper into the bulk. However, in the present study the lattice parameters (lateral interatomic distance and interlayer spacing as well) are kept fixed at their ideal values. Upon a tip-induced surface relaxation, total energy of the tip forces are expected to be lowered. The elastic deformation induced by the tip is local, and hence the forces are expected to penetrate deep into the bulk causing large vertical deformations. ${ }^{9}$ The calculated values in Table I indicate that the small $h$ is a nonequilibrium state, and $E_{T}$ is lowered and the repulsive tip force is decreased with increased $h$. For example, the force between the tip and the surface is still repulsive for $h=4.7$ a.u., but its magnitude is significantly reduced. Further retracting of the tip ( $h=7.6$ a.u.) causes the magnitude of the force to diminish. Defining the potential energy as the difference of the total energies corresponding to the finite and infinite $h$,

$$
V_{T}(h)=E_{T}(h)-E_{T}(h=\infty)
$$

the zero force configuration at $h=7.6$ a.u. is seen to correspond to the minimum of $V_{T}(h)$. Further increase of $h$ beyond this point leads the tip to enter into the attractive

TABLE I. Calculated total energies $\left(E_{T}\right)$ and repulsive tip forces on the tip $\left(F_{T}\right)$ for varying $h$ above the $B$ site. The error bar due to the SCF-convergence criterion in calculated force values is \pm 0.08 mdyn $\left(1 \mathrm{mdyn}=10^{-8} \mathrm{~N}\right)$.

\begin{tabular}{cccc}
\hline & $h=2.7$ a.u. & $h=4.7$ a.u. & $h=7.6$ a.u. \\
\hline$E_{T}(\mathrm{Ry})$ & -74.080 & -74.278 & -74.292 \\
$F_{T}$ (mdyn) & 1.49 & 0.18 & 0.0 \\
\hline \hline
\end{tabular}


region. Soler, Baro, Garcia, and Rohrer ${ }^{9}$ used an empirical potential for $V_{T}(h)$, which was fitted to the elastic constants of the graphite. They predicted that the tip goes from the repulsive to attractive region at $h=6.3$ a.u.

We performed similar calculations for the tip being above the $H$ site with varying $h$. The interatomic forces obtained from these calculations are listed in Table II. For $h=2.7$ a.u. the tip force at the $B$ site is much larger than that at the $H$ site. This situation is, however, reversed when $h$ is increased to 4.7 a.u., where the tip force at the $H$ site becomes larger than that at the $B$ site. On the other hand, the zero force (tip entering into attractive region) of the $H$ site occurs at $h \geq 6.7$ a.u. This implies a zero force corrugation between the $B$ and $H$ sites smaller than 0.9 a.u. It should, however, be noted that owing to the relatively small slope of $F_{T}(h)$ near the boundary between repulsive and attractive force regions the calculated values of $h$ corresponding to $F_{T}(h)=0$ involve a large error bar. Within the calculational parameters and the model used in this study this behavior of the tip force suggests the following explanation: At small $h$, the tip at $B$ site sees higher, but rapidly decaying, surface charge density $^{5}$ relative to the $H$ site. In the region say $4 \leq h \leq 5$ a.u., the core-core repulsion force dominates the tip force, which is large at the $H$ site. Accordingly, as compared to the $H$ site, the tip force at the $B$ site has large value and large decay constant for small $h$, but small decay constant for large $h$. Since the surface charge density calculated within the repeating slab model is lacking reasonable accuracy for $h \geq 5.5$ a.u., the calculated small forces in this region have large error bars.

In conclusion, by using a simple model we have studied the charge density, energetics, and interatomic forces of a system consisting of a three-layer graphite slab and a periodic tip. The most striking result of this study is that as $h$ decreases the electronic states of the graphite and the
TABLE II. Calculated total energies and repulsive tip forces with varying $h$ above the $H$ site. See Table I for further details.

\begin{tabular}{cccr}
\hline \hline & $h=2.7$ a.u. & $h=4.7$ a.u. & $h=6.7$ a.u. \\
\hline$E_{T}$ (Ry) & -74.094 & -74.256 & -74.292 \\
$F_{T}$ (mdyn) & 0.51 & 0.26 & 0.005 \\
\hline \hline
\end{tabular}

tip start to interact, and form localized states. The localization and the energy location of these tip-induced states depend on the type and the position of the tip atom, and undergo a gradual change as $h$ varies. However, their effect on the tunneling current may become almost sudden when their energies cross the Fermi level at a well-defined $h$ and bias voltage (producing a resonant tunneling regime). At this particular tip-to-surface distance they are expected to make a significant contribution to the tunneling current. Marti ${ }^{21}$ has measured the tunneling current from graphite at low temperature as a function of the tip height from the sample. He noted that occasionally current changed by up to an order of magnitude within an $0.1-\AA$ change in the tip height. We take this as the evidence for the collapse of the barrier and a transition to a chemisorption-type state shown in Fig. 1(a). Because of this tip-induced state the STM images, which under conventional circumstances are related to the local density of states at the Fermi level of the clean surface, are strongly affected. Depending on the tip position, not only the total surface charge density, but also the core-core repulsion play a crucial role in determining the tip forces.

The authors wish to acknowledge fruitful discussions with A. Baratoff, G. Binnig, Ch. Gerber, O. J. Marti, H. Rohrer, S. Chiang, M. McClelland, C. F. Quate, R. J. Wilson, H. Salemink, and E. Stoll.
*Permanent address: Department of Physics, Bilkent University, Ankara, Turkey.

${ }^{1}$ G. Binnig, H. Rohrer, Ch. Gerber, and E. Weibel, Phys. Rev. Lett. 49, 57 (1982); 50, 120 (1983).

2 J. Bardeen, Phys. Rev. Lett. 6, 57 (1961); C. B. Duke, in Tunneling in Solids, Solid State Physics, Suppl. 10, edited by H. Ehrenreich, F. Seitz, and D. Turnbull (Academic, New York, 1969).

${ }^{3}$ J. Tersoff and D. R. Hamann, Phys. Rev. Lett. 50, 1998 (1983); Phys. Rev. B 31, 805 (1985).

${ }^{4}$ C. A. Selloni, P. Carnevalli, G. D. Chen, and E. Tosatti, Phys. Rev. B 31, 2602 (1985).

${ }^{5}$ I. P. Batra, N. Garcia, H. Rohrer, H. Salemink, E. Stoll, and S. Ciraci, Surf. Sci. 181, 126 (1987); H. Salemink, I. P. Batra, H. Rohrer, E. Stoll, and E. Weibel, Surf. Sci. 181, 139 (1987); I. P. Batra, S. Ciraci, N. Garcia, H. Rohrer, H. Salemink, and E. Stoll, in Proceedings of the Eighteenth International Conference on the Physics of Semiconductors, Stockholm, 1986, edited by O. Engstrom (World Scientific, Singapore, 1986), p. 57.

${ }^{6}$ D. Tomanek, S. G. Louie, H. J. Mamin, D. W. Abraham, R. E. Thomson, E. Ganz, and J. Clarke, Phys. Rev. B 35, 7790
(1987); H. A. Mizes and W. A. Harrison, in Proceedings of the Second Conference on Scanning Tunneling Microscopy/Spectroscopy [J. Vac. Sci. Technol. A (to be published)].

${ }^{7}$ G. Binnig, H. Fuchs, Ch. Gerber, H. Rohrer, E. Stoll, and E. Tosatti, Europhys. Lett. 1, 31 (1985).

${ }^{8}$ Park Sang-Il and C. F. Quate, Appl. Phys. Lett. 48, 112 (1986); C. F. Quate, Phys. Today 39 (No. 2), 26 (1986).

9 J. M. Soler, A. M. Baro, N. Garcia, and H. Rohrer, Phys. Rev. Lett. 57, 444 (1986)

${ }^{10}$ J. Tersoff, Phys. Rev. Lett. 57, 440 (1986); J. B. Pethica, ibid. 57, 3235 (1986).

${ }^{11}$ H. J. Mamin, E. Ganz, D. W. Abraham, R. E. Thomson, and J. Clarke, Phys. Rev. B 34, 9015 (1986).

${ }^{12}$ G. Binnig, Ch. Gerber, E. Stoll, T. Albrecht, and C. F. Quate, Europhys. Lett. (to be published).

${ }^{13}$ G. Binnig, C. F. Quate, and Ch. Gerber, Phys. Rev. Lett. 56, 930 (1986).

${ }^{14}$ M. Schluter, J. R. Chelikowsky, S. G. Louie, and M. L. Cohen, Phys. Rev. B 12, 4200 (1975).

15 J. Ihm, A. Zunger, and M. L. Cohen, J. Phys. C 12, 4409 (1979). 
${ }^{16} \mathrm{Graphite}$ surface has two different atomic sites. The $B$ site lies above the center of the second-layer hexagram. The $A$ site has a second-layer atom directly below. The $H$ site denotes the center of the surface hexagram.

${ }^{17}$ R. C. Tatar and S. Rabii, Phys. Rev. B 25, 4126 (1982); I. P. Batra and L. Samuelson, Synth. Met. 1, 223 (1979/80).

${ }^{18}$ M. T. Yin and M. L. Cohen, Phys. Rev. B 33, 4294 (1986).

${ }^{19}$ I. P. Batra, S. Ciraci, G. P. Srivastava, J. S. Nelson, and C. Y.
Fong, Phys. Rev. B 34, 8246 (1986); I. P. Batra, S. Ciraci, and J. S. Nelson, J. Vac. Sci. Technol. B 5, 1300 (1987).

${ }^{20}$ See, for example, P. Bendt and A. Zunger, Phys. Rev. Lett. 50, 1684 (1983).

${ }^{21}$ O. J. Marti, Ph. D. thesis, Eidgenössische Technische Hochschule Zürich, 1986 (unpublished); O. J. Marti and H. Rohrer, Bull. Am. Phy. Soc. 32, 524 (1987). 